\title{
Aberrations in Oxidative Stress Markers in Amyotrophic Lateral Sclerosis: A Systematic Review and Meta-Analysis
}

\author{
Zihao Wang, Zhile Bai, Xiaoyan Qin $\left(\mathbb{D}\right.$, and Yong Cheng $\mathbb{D}^{\mathrm{D}}$ \\ Key Laboratory of Ethnomedicine for Ministry of Education, Center on Translational Neuroscience, College of Life and \\ Environmental Sciences, Minzu University of China, Beijing 100081, China \\ Correspondence should be addressed to Xiaoyan Qin; bjqinxiaoyan@muc.edu.cn and Yong Cheng; yongcheng@muc.edu.cn
}

Received 1 March 2019; Accepted 20 May 2019; Published 9 June 2019

Guest Editor: Giovanna Cenini

Copyright (c) 2019 Zihao Wang et al. This is an open access article distributed under the Creative Commons Attribution License, which permits unrestricted use, distribution, and reproduction in any medium, provided the original work is properly cited.

\begin{abstract}
Oxidative stress has been reported to be involved in the onset and development of amyotrophic lateral sclerosis (ALS). Data from clinical studies have highlighted increased peripheral blood oxidative stress markers in patients with ALS, but results are inconsistent. Therefore, we quantitatively pooled data on levels of blood oxidative stress markers in ALS patients from the literature using a meta-analytic technique. A systematic search was performed on PubMed and Web of Science, and we included studies analyzing blood oxidative stress marker levels in patients with ALS and normal controls. We included 41 studies with 4,588 ALS patients and 6,344 control subjects, and 15 oxidative stress marker levels were subjected to randomeffects meta-analysis. The results demonstrated that malondialdehyde (Hedges' $g, 1.168 ; 95 \% \mathrm{CI}, 0.812$ to $1.523 ; P<0.001$ ), 8-hydroxyguanosine (Hedges' $g, 2.194 ; 95 \%$ CI, 0.554 to 3.835; $P=0.009$ ), and Advanced Oxidation Protein Product (Hedges' $g, 0.555 ; 95 \%$ CI, 0.317 to $0.792 ; P<0.001$ ) levels were significantly increased in patients with ALS when compared with control subjects. Uric acid (Hedges' $g,-0.798$; 95\% CI, -1.117 to $-0.479 ; P<0.001$ ) and glutathione (Hedges' $g,-1.636$; 95\% CI, -3.020 to $-0.252 ; P=0.02$ ) levels were significantly reduced in ALS patients. In contrast, blood Cu, superoxide dismutase, glutathione peroxidase, ceruloplasmin, triglycerides, total cholesterol, low-density lipoprotein, high-density lipoprotein, coenzyme-Q10, and transferrin levels were not significantly different between cases and controls. Taken together, our results showed significantly increased blood levels of 8-hydroxyguanosine, malondialdehyde, and Advanced Oxidation Protein Product and decreased glutathione and uric acid levels in the peripheral blood of ALS patients. This meta-analysis helps to clarify the oxidative stress marker profile in ALS patients, supporting the hypothesis that oxidative stress is a central component underpinning ALS pathogenesis.
\end{abstract}

\section{Introduction}

Amyotrophic lateral sclerosis (ALS) is a devastating neurodegenerative disease caused by cell death of both upper and lower motor neurons [1]. It is known that $90-95 \%$ of cases are sporadic and the remaining $5-10 \%$ of cases are due to genetic predisposition [2]. The onset of ALS usually begins at the age of 50 years old for genetically inherited cases and at the age of 60 years old for sporadic cases, although the disease can start at any age [2]. It is estimated that two to three people out of 100,000 are affected by ALS in the United States and Europe every year [3,4], while the incidence of the disease in the rest of the world remains largely unknown. Due to the poorly understood etiology of ALS, there is no effective treatment for the disease, and most patients survive between 2 to 4 years after diagnosis [5]. FDA has approved two drugs (riluzole and edaravone) for the treatment of ALS, and riluzole is reported to increase the life expectancy of patients by 2 to 3 months [6]. Therefore, it is critical to elucidate the etiology of ALS to facilitate the development of novel therapies for this devastating disease.

Although little is known regarding the cause of ALS, accumulating evidence suggests that increased inflammatory responses and oxidative stress alongside glial cell dysfunction play crucial roles in disease pathogenesis; this is supported by clinical studies showing infiltration of immune cells and heightened inflammatory cytokine profiles in the central nervous system of ALS patients [7, 8]. Although cytokine data 
are inconsistent across studies, a meta-analysis by Chen et al. reported that granulocyte-colony stimulating factor, interleukin-2 (IL-2), IL-15, IL-17, monocyte chemotactic protein-1, macrophage inflammatory protein-1 $\alpha$, tumor necrosis factor- $\alpha$ (TNF- $\alpha$ ), and vascular endothelial growth factor levels in the cerebrospinal fluid were significantly elevated in patients with ALS when compared with controls [9]. Another meta-analysis performed by our group including 25 studies clarified the peripheral blood inflammatory cytokine profile in ALS, which revealed elevated blood TNF- $\alpha$, TNF receptor 1, IL-6, IL-1 $\beta$, IL-8, and vascular endothelial growth factor levels in patients with ALS relative to control subjects [10]. In addition, a substantial number of clinical studies have analyzed oxidative stress markers in ALS and demonstrated that levels of prooxidative stress markers, malondialdehyde (MDA) and 8-hydroxyguanosine $(8-\mathrm{OHdG})$ were increased in the peripheral blood of ALS patients [11, 12]. In contrast, decreased antioxidant glutathione and uric acid levels were observed in ALS patients [13, 14]. However, other studies have reported unaltered levels of antioxidants in patients with ALS compared to controls $[15,16]$. Due to the heterogeneity of the clinical data on oxidative stress markers, the profile of oxidative stress markers in ALS patients remains unclear.

To better understand the etiology of ALS and potentially use oxidative stress markers for the diagnosis and prognosis of ALS patients, we reviewed PubMed and Web of Science systematically and pooled the data from the included studies to clarify the oxidative stress marker profile in patients with ALS.

\section{Materials and Methods}

This systematic review and meta-analysis was performed according to the instructions that are recommended by the PRISMA statement (Preferred Reporting Items for Systematic Reviews and Meta-Analysis) [17].

2.1. Literature Search. Two independent investigators manually reviewed English-language articles on PubMed and Web of Science from May 2018 to September 2018. The search terms for the systematic review were the following: (oxidative stress or catalase or hydroxyguanosine or malondialdehyde or uric acid or ceruloplasmin or glutathione or transferrin or low density lipoprotein or copper or cholesterol) and amyotrophic lateral sclerosis. We included original articles that reported peripheral blood levels of oxidative stress markers in ALS patients and control subjects.

2.2. Data Extraction. We extracted sample size, mean oxidative stress marker concentrations, $P$ values, and standard deviation (s.d.) as the primary outcomes for this metaanalysis. We also extracted additional data on age, gender (proportion of males), publication year, sampling source, disease duration, and diagnosis of potential moderator analyses. The demographic and clinical variables of the included studies in this meta-analysis are presented in Supplementary Table (available here).
2.3. Statistical Analysis. The Comprehensive Meta-Analysis Version 2 software (Biostat, Englewood, NJ, USA) was used to pool the oxidative stress marker data on ALS patients. The sample size, mean oxidative stress marker concentration, and s.d. were primarily used to generate the effective size (ES). Sample size and $P$ values were used to generate ES if oxidative stress marker concentration data were not available. An ES was calculated as the standardized mean difference in oxidative stress marker concentrations between cases and controls and then converted to Hedge's $g$ which provides ES adjustment for sample size [18]. The 95\% confidence interval $(95 \% \mathrm{CI})$ was used to estimate the statistical significance of the pooled ES. We performed randomeffects meta-analysis for this study because we estimated that the true ES would be affected by between-study and within-study variations [19]. We also performed sensitivity analysis by removing one study at a time to evaluate whether the statistical significance between cases and controls for oxidative stress marker concentrations was influenced by a single study.

The statistical difference of between-study heterogeneity was evaluated using the Cochran $Q$ test [20], whereby statistical significance was set at $P$ value $<0.1$. The impact of between-study heterogeneity was evaluated by the $I^{2}$ index, and $I^{2}$ of $0.25,0.50$, and 0.75 suggested small, medium, and high levels of heterogeneity, respectively. We then used unrestricted maximum-likelihood random-effects metaregressions of ESs to analyze whether age, gender, or publication year had moderating effects on the outcomes of the metaanalysis. The publication bias for the included studies in this meta-analysis was determined by Egger's test [21], which assesses the degree of funnel plot asymmetry. The statistical significance of this meta-analysis was set at $P$ value $<0.05$ unless stated otherwise.

\section{Results}

Our initial search with the keywords produced 2,120 records from PubMed and 2,162 records from Web of Science. After screening the titles and abstracts of the records, 146 articles were selected for full-text scrutiny. Of the 146 studies, 105 studies were excluded due to the following reasons: no necessary data $(n=77)$; without a control group $(n=8)$; oxidative stress markers were analyzed in less than two studies $(n=7)$; studies were single case reports $(n=4)$; studies were review articles $(n=5)$; or data overlapped with other studies $(n=4)$. A final total of 41 articles with 4,588 ALS patients and 6,344 controls were included in this meta-analysis [11-16, 22-56] (for the flowchart, see Figure 1).

3.1. Main Associations of Blood Oxidative Stress Markers with ALS. Results from the meta-analysis showed that blood MDA (Hedges' $g, 1.168$; 95\% CI, 0.812 to 1.523 ; $P<0.001$ ), 8-OhDG (Hedges' $g, 2.194 ; 95 \% \mathrm{CI}, 0.554$ to 3.835; $P=0.009)$, and Advanced Oxidation Protein Product (AOPP, Hedges' $g$, 0.555; 95\% CI, 0.317 to $0.792 ; P<0.001$ ) levels were significantly elevated in patients with ALS when compared with controls, whereas blood uric acid (Hedges' $g$, -0.798 ; $95 \%$ CI, -1.117 to $-0.479 ; P<0.001)$ and glutathione 

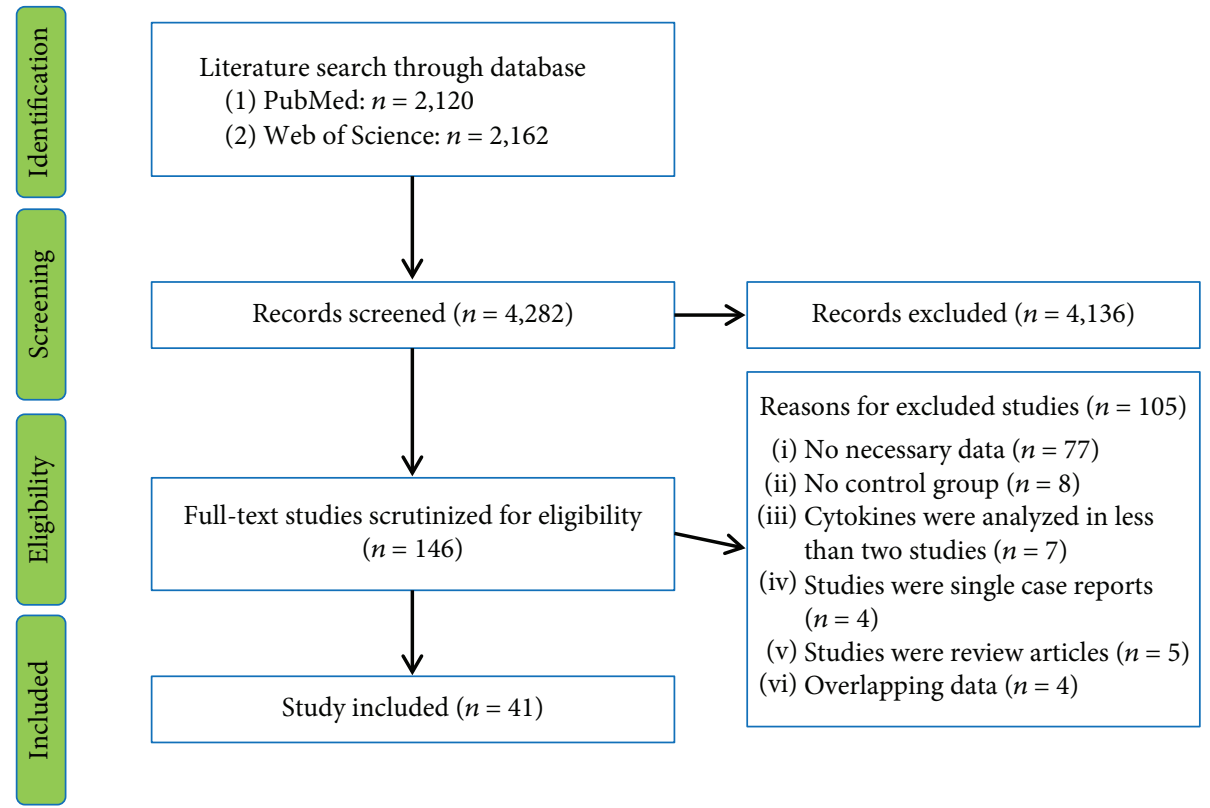

FIgURE 1: PRISMA flowchart of the literature search.

(Hedges' $g,-1.636$; 95\% CI, -3.020 to $-0.252 ; P=0.02$ ) levels were significantly decreased in ALS patients (Figures 2 and 3 and Table 1). In addition, we did not observe significant differences between ALS patients and controls for blood $\mathrm{Cu}$, superoxide dismutase (SOD), glutathione peroxidase, coenzyme-Q10 (Co-Q10), ceruloplasmin, total cholesterol, triglycerides, high-density lipoprotein (HDL), low-density lipoprotein (LDL), and transferrin levels (Table 1).

3.2. Investigation of Heterogeneity. For the fifteen oxidative stress markers analyzed in the meta-analysis, AOPP and ceruloplasmin did not show between-study heterogeneity. MDA showed small levels of between-study heterogeneity, Co-Q10 showed moderate levels of between-study heterogeneity, and high levels of heterogeneity among studies were found for the other eleven markers (Table 1).

We next explored whether potential moderators accounted for the heterogeneity for the ALS-associated five oxidative stress markers. Given that blood MDA and AOPP showed low levels of between-study heterogeneity, and due to the limited number of studies with small size analyzing 8-OHdG and glutathione levels, we performed metaregression and subgroup analyses on uric acid. As shown in the Supplementary Table, the information on disease duration of ALS patients was limited. We therefore conducted metaregression analyses according to age, gender, and publication year. Metaregression analyses suggested that publication year, gender, and age did not significantly affect the results of the meta-analysis ( $P>0.05$ in all analyses).

Next, we performed subgroup analyses based on the sampling source. Uric acid levels were reduced both in the serum (Hedges' $g=-0.810,95 \% \mathrm{CI}=-1.190$ to $-0.431, P<0.001$ ) and the plasma (Hedges' $g=-0.764,95 \% \mathrm{CI}=-1.380$ to $0.148, P=0.015)$ of ALS patients when compared with those of the controls. However, between-study heterogeneity was increased for the serum studies $(Q=53.542 ; \mathrm{df}=4$; $\left.I^{2}=92.529 ; P<0.001\right)$ but reduced for the plasma studies $\left(Q=2.478 ;\right.$ df $\left.=1 ; I^{2}=59.643 ; P=0.115\right)$.

Furthermore, we performed sensitivity analysis and showed that the significant association between blood uric acid and ALS was not influenced by any individual study.

Inspection of funnel plots visually indicated no publication bias for studies analyzing uric acid or MDA levels in ALS patients (Figure 4); these were confirmed by the Egger test (Table 1, $P>0.1$ ). However, the funnel plot suggested there may be publication bias for studies analyzing glutathione levels in ALS patients (Figure 4). Further, the Egger test suggested a trend for publication bias for glutathione (Table $1, P=0.061$ ). To analyze the effect of potential publication bias, we used the classic fail-safe $\mathrm{N}^{18}$ to compute the number of missing studies (with mean effect of zero) that would require to bring the $P$ value above 0.05 for glutathione, and the analysis showed that 30 studies would need to be added to generate a nonsignificant association between glutathione and ALS, suggesting that potential publication bias is unlikely to significantly affect the positive outcome of the present study. Due to the limited number of studies, we were unable to perform publication bias analysis for $8-\mathrm{OHdG}$ and AOPP.

\section{Discussion}

This meta-analysis included 41 studies with 4,588 ALS patients and 6,344 control individuals analyzing 15 oxidative stress markers from the blood. The results suggest that MDA (the important end product for lipid peroxidation), 8-OhdG (a marker for DNA damage), and AOPP were significantly elevated in the blood of ALS patients when compared with control individuals. In addition, we found that the levels of antioxidant glutathione and uric acid were significantly 
8-OHdG

\begin{tabular}{lcccc} 
Study name & \multicolumn{5}{c}{ Statistics for each study } \\
& Hedges' Lower & Upper & \\
& $g$ & limit & limit & $P$ value \\
Kihira et al. 2013 & 3.020 & 2.136 & 3.904 & 0.000 \\
Blasco et al. 2016 & 1.346 & 0.385 & 2.307 & 0.006 \\
Overall & 2.194 & 0.554 & 3.835 & 0.009
\end{tabular}

\begin{tabular}{ccccr} 
Study name & \multicolumn{5}{c}{ Statistics for each study } \\
& Hedges' & Lower & Upper & \\
& $g$ & limit & limit & $P$ value \\
Kihira et al. 2013 & 3.020 & 2.136 & 3.904 & 0.000 \\
Blasco et al. 2016 & 1.346 & 0.385 & 2.307 & 0.006 \\
Overall & 2.194 & 0.554 & 3.835 & 0.009
\end{tabular}

(a)

GSH



(b)

AOPP

\begin{tabular}{lcccc} 
Study name & \multicolumn{3}{c}{ Statistics for each study } \\
& Hedges' Lower & Upper & \\
$g$ & limit & limit & $P$ value \\
Pasquinelli et al. 2016 & 0.574 & 0.236 & 0.912 & 0.001 \\
LoGerfo et al. 2014 & 0.536 & 0.201 & 0.870 & 0.002 \\
$\quad$ Overall & 0.555 & 0.317 & 0.792 & 0.000
\end{tabular}

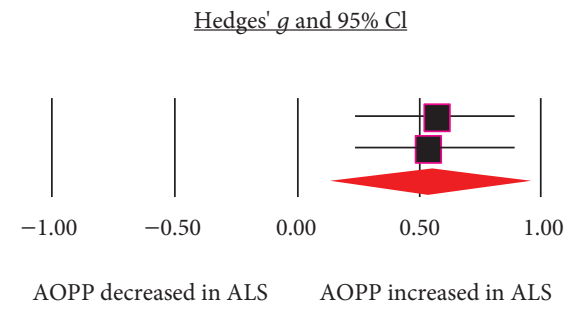

(c)

Figure 2: Studies of blood 8-OHdG, GSH, and AOPP in amyotrophic lateral sclerosis. Forest plot displaying random-effects meta-analysis results of the association between 8-OHdG (a), GSH (b), AOPP (c), and amyotrophic lateral sclerosis. GSH: glutathione; AOPP: Advanced Oxidation Protein Product; 8-OHdG: 8-hydroxyguanosine.

downregulated in patients with ALS. However, other oxidative stress markers including $\mathrm{Cu}, \mathrm{SOD}$, glutathione peroxidase, ceruloplasmin, triglycerides, total cholesterol, LDL, HDL, Co-Q10, and transferrin were not significantly associated with ALS. For the five dysregulated oxidative stress markers in ALS patients, the results associated with ESs of 8-OHdG, MDA, and GSH were large, and the ESs were medium to large for AOPP and uric acid. Sensitivity analysis indicated that no individual study influenced the significantly decreased blood uric acid levels in ALS patients, and no publication bias risks were observed for studies analyzing uric acid and MDA concentrations as determined by funnel plots and the Egger test, indicating the robustness of the results from our present study.

Although our study is the first to use a meta-analytic technique to clarify the oxidative stress marker profile in patients with ALS, it is unclear whether oxidative stress has causal effect for ALS onset and/or development. However, the important role of oxidative stress in the pathogenesis of ALS is supported by the fact that the mutations in the gene encoding the cytosolic antioxidant enzyme-SOD1 cause ALS [57]. In addition, mutant SOD1 transgenic mice exhibited age-dependent motor neuron degeneration accompanied by the biochemical changes in the nerve cells [58]. Moreover, it has been reported that uric acid levels were negatively correlated with the disease progression in ALS patients [44]. Collectively, these previous findings and our pooled clinical data of the dysfunction between the oxidation and antioxidant systems in ALS patients support the hypothesis that oxidative stress is central in the pathogenesis of ALS.

Our analyses further showed that most of the oxidative stress markers had high levels of heterogeneity among studies. However, for the five oxidative stress markers that were dysregulated in the patients with ALS, AOPP did not show between-study heterogeneity and MDA showed small levels of heterogeneity, suggesting the reproducibility of these results. In addition, we conducted subgroup and metaregression analyses to address moderators that may explain heterogeneity for uric acid. The results indicated that gender, age, and publication year did not contribute to the between- 
Uric acid

$\underline{\text { Study name }}$

Ikeda et al. 2009

Oh et al. 2015

Zheng et al. 2014

Zoccolella et al. 2010

Keizman et al. 2009

Sohmiya et al. 2005

Nagase et al. 2016

Overall $\underline{\text { Statistics for each study }}$

$\begin{array}{cccc}\begin{array}{c}\text { Hedges' } \\ g\end{array} & \begin{array}{l}\text { Lower } \\ \text { limit }\end{array} & \begin{array}{l}\text { Upper } \\ \text { limit }\end{array} & P \text { value } \\ -1.240 & -1.668 & -0.813 & 0.000 \\ -0.843 & -1.090 & -0.595 & 0.000 \\ -1.067 & -1.199 & -0.936 & 0.000 \\ -0.202 & -0.404 & -0.001 & 0.049 \\ -0.762 & -1.070 & -0.454 & 0.000 \\ -0.420 & -1.034 & 0.195 & 0.180 \\ -1.051 & -1.540 & -0.561 & 0.000 \\ -0.798 & -1.117 & -0.479 & 0.000\end{array}$

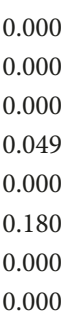

Study name

Moumen et al. 1997

Baillet et al. 2010

Blasco et al. 2010

Oteiza et al. 1997

Rousselot et al. 2000

Overall $\underline{\text { Statistics for each study }}$

$\begin{array}{ccc}\text { Hedges' } & \text { Lower } & \text { Upper } \\ g & \text { limit } & \text { limit }\end{array}$

$\begin{array}{lll}2.038 & 1.051 & 3.026\end{array}$

$1.355 \quad 0.804 \quad 1.906$

$\begin{array}{lll}1.226 & 0.282 & 2.170\end{array}$

$\begin{array}{lll}0.705 & -0.039 & 1.449\end{array}$

$\begin{array}{lll}0.968 & 0.560 & 1.379\end{array}$

$\begin{array}{lll}1.168 & 0.812 \quad 1.523\end{array}$ $\mid$

Uric acid decreased in ALS Uric acid increased in ALS

(a)

MDA



\section{Hedges' $g$ and $95 \% \mathrm{Cl}$}

Hedges' $g$ and $95 \% \mathrm{Cl}$

value
0.000
0.000
0.011
0.063
0.000
0.000

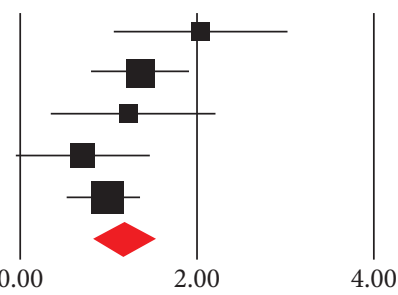

MDA increased in ALS

(b)

FIGURE 3: Studies of blood uric acid and MDA in amyotrophic lateral sclerosis. Forest plot displaying random-effects meta-analysis results of the association between uric acid (a), MDA (b), and amyotrophic lateral sclerosis. MDA: malondialdehyde.

study heterogeneity. Although subgroup analyses based on sampling source revealed that between-study heterogeneity was reduced in plasma studies analyzing uric acid levels, the lower heterogeneity is likely due to the low power of the test for heterogeneity used in meta-analyses with smaller numbers of studies. Other clinical variables including medication status and disease duration may also contribute to between-study heterogeneity. However, most of the studies included in the meta-analysis did not provide this information, thus preventing us from performing subgroup or metaregression analyses to assess whether these factors contributed to between-study heterogeneity. Indeed, a study reported that lithium and valproate cotreatment increased the survival of patients with ALS and the treatment also significantly increased blood glutathione levels in these patients [24].

The second limitation of this study is how much the alterations in oxidative stress markers in the peripheral blood reflect changes in the central nervous system. However, Djordjevic et al. reported that patients with ALS had significantly increased cerebrospinal fluid AOPP levels relative to control subjects [59]. Moreover, Murata et al. demonstrated that ALS patients had higher CSF 8-OHdG concentrations than control subjects [60]. These results support the "peripheral as a window to the brain" hypothesis. Further studies are necessary to translate these findings into practical clinical use. The third limitation of this study is that some oxidative stress markers analyzed in this study such as glutathione peroxidase had a limited number of studies with small sample sizes; therefore, it is difficult to determine significant associations between these markers and ALS. In addition, several other important oxidative stress markers such as catalase were not analyzed in the meta-analysis due to the lack of clinical studies on these markers. Future studies should clarify the role of oxidative stress in the onset and development of ALS.

In addition to prooxidative stress imbalance observed in ALS, a large number of studies have measured oxidative stress markers in other neurodegenerative diseases including Alzheimer's disease [61] and Parkinson's disease [62]. Due to the heterogeneous etiologies of these diseases, it is not surprising that results are inconsistent across studies comparing oxidative stress marker levels between patients with Alzheimer's disease or Parkinson's disease and controls. To address the inconsistent clinical data, Schrag et al. performed 
TABLE 1: Summary of comparative outcomes for measurements of blood oxidative stress marker levels.

\begin{tabular}{|c|c|c|c|c|c|c|c|c|c|c|c|}
\hline \multirow[b]{2}{*}{ Cytokine } & \multirow{2}{*}{$\begin{array}{l}\text { No. of } \\
\text { studies }\end{array}$} & \multirow{2}{*}{$\begin{array}{c}\text { No. with } \\
\text { ALS/controls }\end{array}$} & \multicolumn{2}{|l|}{ Main effect } & \multirow[b]{2}{*}{$\begin{array}{c}P \\
\text { value }\end{array}$} & \multicolumn{4}{|c|}{ Heterogeneity } & \multicolumn{2}{|c|}{ Publication bias } \\
\hline & & & Hedges' $g(95 \%$ CI $)$ & $\begin{array}{c}z \\
\text { score }\end{array}$ & & $\begin{array}{c}Q \\
\text { statistic }\end{array}$ & $\mathrm{df}$ & $\begin{array}{c}P \\
\text { value }\end{array}$ & $\begin{array}{c}I^{2} \\
\text { statistic }\end{array}$ & $\begin{array}{c}\text { Egger } \\
\text { intercept }\end{array}$ & $P$ value \\
\hline Uric acid & 7 & $961 / 1185$ & $-0.798(-1.117$ to -0.479$)$ & -4.906 & $<0.001$ & 56.034 & 6 & $<0.001$ & 89.292 & 0.910 & 0.750 \\
\hline 8-OHdG & 2 & $18 / 61$ & $2.194(0.554$ to 3.835$)$ & 2.622 & 0.009 & 6.315 & 1 & 0.012 & 84.165 & NA & NA \\
\hline MDA & 5 & $123 / 120$ & $1.168(0.812$ to 1.523$)$ & 6.441 & $<0.001$ & 5.793 & 4 & 0.215 & 30.950 & 1.61721 & 0.38172 \\
\hline GSH & 4 & $71 / 78$ & $-1.636(-3.020$ to -0.252$)$ & -2.318 & 0.020 & 36.112 & 3 & $<0.001$ & 91.692 & -7.014 & 0.061 \\
\hline AOPP & 2 & $147 / 133$ & $0.555(0.317$ to 0.792$)$ & 4.571 & $<0.001$ & 0.025 & 1 & 0.875 & 0.000 & NA & NA \\
\hline Ceruloplasmin & 3 & $46 / 46$ & $-0.052(-0.475$ to 0.372$)$ & -0.239 & 0.811 & 2.168 & 2 & 0.338 & 7.742 & 2.538 & 0.403 \\
\hline $\mathrm{Cu}$ & 10 & $361 / 471$ & $0.014(-0.337$ to 0.366$)$ & 0.080 & 0.937 & 39.670 & 9 & $<0.001$ & 77.313 & 0.09686 & 0.94797 \\
\hline $\begin{array}{l}\text { Glutathione } \\
\text { peroxidase }\end{array}$ & 4 & $91 / 110$ & $-0.679(-1.732$ to 0.373$)$ & -1.265 & 0.206 & 29.687 & 3 & $<0.001$ & 89.895 & -4.92304 & 0.30857 \\
\hline Total cholesterol & 13 & $2161 / 3870$ & $0.144(-0.095$ to 0.383$)$ & 1.184 & 0.236 & 160.185 & 12 & $<0.001$ & 92.509 & 1.50578 & 0.44161 \\
\hline HDL & 9 & $2024 / 3722$ & $-0.198(-0.625$ to 0.228$)$ & -0.912 & 0.362 & 345.128 & 8 & $<0.001$ & 97.682 & 2.99958 & 0.54140 \\
\hline LDL & 9 & $2024 / 3722$ & $0.121(-0.283$ to 0.524$)$ & 0.587 & 0.557 & 307.948 & 8 & $<0.001$ & 97.402 & 2.74193 & 0.55440 \\
\hline SOD & 5 & $96 / 184$ & $-0.203(-0.904$ to 0.497$)$ & -0.569 & 0.569 & 22.703 & 4 & $<0.001$ & 82.381 & 9.11205 & 0.01197 \\
\hline Co-Q10 & 4 & $95 / 128$ & $0.040(-0.468$ to 0.548$)$ & 0.156 & 0.876 & 9.953 & 3 & 0.019 & 69.858 & -2.10679 & 0.75503 \\
\hline Transferrin & 5 & $954 / 592$ & $0.244(-0.219$ to 0.707$)$ & 1.032 & 0.302 & 50.330 & 4 & $<0.001$ & 92.052 & 5.24433 & 0.09393 \\
\hline Triglyceride & 8 & $1743 / 1633$ & $-0.154(-0.622$ to 0.314$)$ & -0.645 & 0.519 & 265.560 & 7 & $<0.001$ & 97.364 & 4.85477 & 0.32384 \\
\hline
\end{tabular}

Abbreviations: df: degrees of freedom; ALS: amyotrophic lateral sclerosis; 8-OHdG: 8-hydroxyguanosine; MDA: malondialdehyde; SOD: superoxide dismutase; AOPP: Advanced Oxidation Protein Product; LDL: low-density lipoprotein; HDL: high-density lipoprotein; Co-Q10: coenzyme-Q10; NA: not available.

Uric acid

Funnel plot of precision by Hedges' $g$

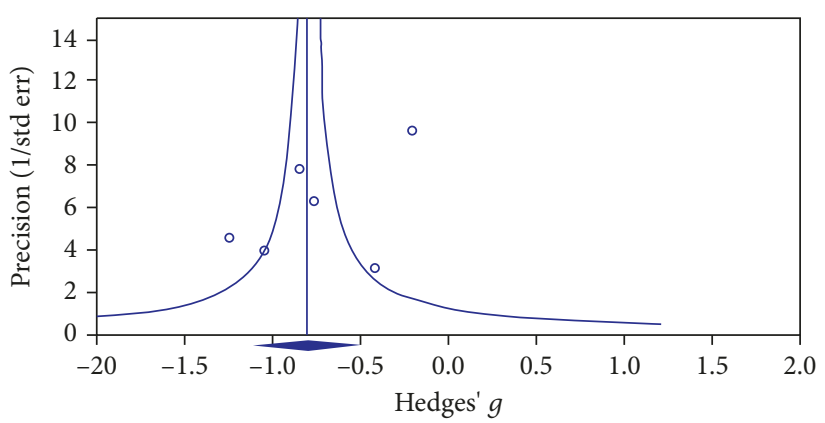

(a)
Glutathione

Funnel plot of precision by Hedges' $g$



(b)

Malondialdehyde

Funnel plot of precision by Hedges' $g$

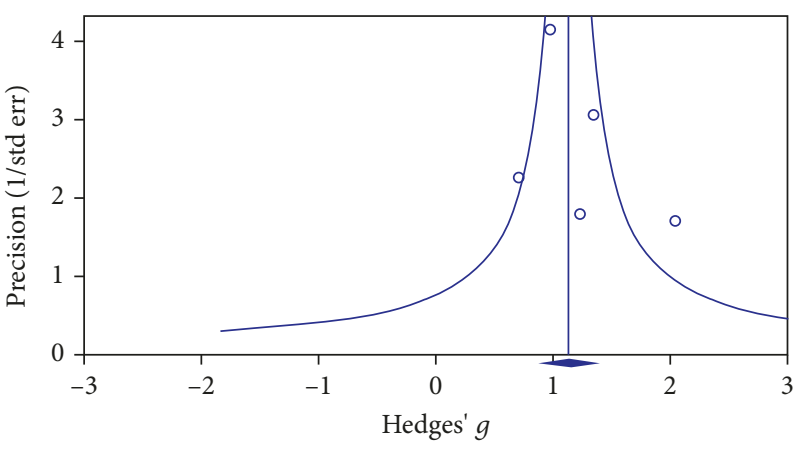

(c)

FIGURE 4: Funnel plots examining publication bias in studies comparing blood uric acid (a), GSH (b), and MDA (c) levels between cases and controls. GSH: glutathione; MDA: malondialdehyde. 
a meta-analysis and reported that patients with Alzheimer's disease were accompanied by reduced uric acid levels and increased MDA levels in the peripheral blood [63]. In addition, a systematic review and meta-analysis performed by Wei et al. showed that blood 8-OHdG and MDA levels were elevated in Parkinson's disease patients, whereas uric acid and glutathione levels were downregulated in these patients [64]. The dysregulated profiles of oxidative stress markers in Parkinson's disease and Alzheimer's disease were similar to the findings from our meta-analysis on profiles of oxidative stress markers in ALS, suggesting a common pathway that confers vulnerability to the development of these neurodegenerative diseases.

In conclusion, the findings from the present study revealed increased 8-OhdG, MDA, and AOPP levels and reduced uric acid and glutathione levels in the peripheral blood of ALS patients. Our results clarify the oxidative stress marker profile in the blood of ALS patients and strengthens the clinical evidence that prooxidative imbalances contribute to ALS pathophysiology.

\section{Data Availability}

The data used to support the findings of this study are included within the article.

\section{Conflicts of Interest}

The authors declare that they have no conflict of interest.

\section{Acknowledgments}

This study was supported by the National Science Foundation of China (81703492), the Beijing Natural Science Foundation (7182092), the Minzu University Research Fund (2018CXTD03), and the MUC 111 project.

\section{Supplementary Materials}

Supplementary Table: characteristics of included studies measuring peripheral blood oxidative stress marker levels. (Supplementary Materials)

\section{References}

[1] W. Huynh, N. G. Simon, J. Grosskreutz, M. R. Turner, S. Vucic, and M. C. Kiernan, "Assessment of the upper motor neuron in amyotrophic lateral sclerosis," Clinical Neurophysiology, vol. 127, no. 7, pp. 2643-2660, 2016.

[2] M. C. Kiernan, S. Vucic, B. C. Cheah et al., "Amyotrophic lateral sclerosis," The Lancet, vol. 377, no. 9769, pp. 942-955, 2011.

[3] O. Hardiman, A. Al-Chalabi, C. Brayne et al., "The changing picture of amyotrophic lateral sclerosis: lessons from European registers," Journal of Neurology, Neurosurgery \& Psychiatry, vol. 88, no. 7, pp. 557-563, 2017.

[4] P. Mehta, V. Antao, W. Kaye et al., "Prevalence of amyotrophic lateral sclerosis-United States, 2010-2011," Morbidity and Mortality Weekly Report Supplements, vol. 63, no. 7, pp. 1-14, 2014.
[5] J. D. Rothstein, "Current hypotheses for the underlying biology of amyotrophic lateral sclerosis," Annals of Neurology, vol. 65, Supplement 1, pp. S3-S9, 2009.

[6] R. G. Miller, J. D. Mitchell, and D. H. Moore, "Riluzole for amyotrophic lateral sclerosis (ALS)/motor neuron disease (MND)," Cochrane Database of Systematic Reviews, vol. 3, 2012.

[7] H. R. Martinez, C. E. Escamilla-Ocanas, C. R. CamaraLemarroy, M. T. Gonzalez-Garza, J. Moreno-Cuevas, and M. A. Garcia Sarreon, "Increased cerebrospinal fluid levels of cytokines monocyte chemoattractant protein-1 (MCP-1) and macrophage inflammatory protein- $1 \beta$ (MIP- $1 \beta)$ in patients with amyotrophic lateral sclerosis," Neurologia, vol. 32, 2017.

[8] W. Zhao, D. R. Beers, and S. H. Appel, "Immune-mediated mechanisms in the pathoprogression of amyotrophic lateral sclerosis," Journal of Neuroimmune Pharmacology, vol. 8, no. 4, pp. 888-899, 2013.

[9] X. Chen, Y. Hu, Z. Cao, Q. Liu, and Y. Cheng, "Cerebrospinal fluid inflammatory cytokine aberrations in Alzheimer's disease, Parkinson's disease and amyotrophic lateral sclerosis: a systematic review and meta-analysis," Frontiers in Immunology, vol. 9, article 2122, 2018.

[10] Y. Hu, C. Cao, X. Y. Qin et al., "Increased peripheral blood inflammatory cytokine levels in amyotrophic lateral sclerosis: a meta-analysis study," Scientific Reports, vol. 7, no. 1, p. 9094, 2017.

[11] H. Blasco, G. Garcon, F. Patin et al., "Panel of oxidative stress and inflammatory biomarkers in ALS: a pilot study," Canadian Journal of Neurological Sciences / Journal Canadien des Sciences Neurologiques, vol. 44, no. 1, pp. 90-95, 2017.

[12] R. Moumen, A. Nouvelot, D. Duval, B. Lechevaliera, and F. Viadera, "Plasma superoxide dismutase and glutathione peroxidase activity in sporadic amyotrophic lateral sclerosis," Journal of the Neurological Sciences, vol. 151, no. 1, pp. 35-39, 1997.

[13] J. Ehrhart, A. J. Smith, N. Kuzmin-Nichols et al., "Humoral factors in ALS patients during disease progression," Journal of Neuroinflammation, vol. 12, no. 1, p. 127, 2015.

[14] K. Ikeda, K. Kawabe, and Y. Iwasaki, "Do serum uric acid levels reflect oxidative stress in the progression of ALS?," Journal of the Neurological Sciences, vol. 287, no. 1-2, p. 294, 2009.

[15] A. Baillet, V. Chanteperdrix, C. Trocme, P. Casez, C. Garrel, and G. Besson, "The role of oxidative stress in amyotrophic lateral sclerosis and Parkinson's disease," Neurochemical Research, vol. 35, no. 10, pp. 1530-1537, 2010.

[16] M. Sohmiya, M. Tanaka, Y. Suzuki, Y. Tanino, K. Okamoto, and Y. Yamamoto, "An increase of oxidized coenzyme Q-10 occurs in the plasma of sporadic ALS patients," Journal of the Neurological Sciences, vol. 228, no. 1, pp. 49-53, 2005.

[17] D. Moher, A. Liberati, J. Tetzlaff, D. G. Altman, and The PRISMA Group, "Preferred reporting items for systematic reviews and meta-analyses: the PRISMA statement," PLoS Medicine, vol. 6, no. 7, article e1000097, 2009.

[18] X. Y. Qin, S. P. Zhang, C. Cao, Y. P. Loh, and Y. Cheng, "Aberrations in peripheral inflammatory cytokine levels in Parkinson disease: a systematic review and meta-analysis," JAMA Neurology, vol. 73, no. 11, pp. 1316-1324, 2016.

[19] Y. Du, H. T. Wu, X. Y. Qin et al., "Postmortem brain, cerebrospinal fluid, and blood neurotrophic factor levels in Alzheimer's disease: a systematic review and meta-analysis," Journal of Molecular Neuroscience, vol. 65, no. 3, pp. 289-300, 2018. 
[20] X. Y. Qin, J. C. Feng, C. Cao, H. T. Wu, Y. P. Loh, and Y. Cheng, "Association of peripheral blood levels of brainderived neurotrophic factor with autism spectrum disorder in children: a systematic review and meta-analysis," JAMA Pediatrics, vol. 170, no. 11, pp. 1079-1086, 2016.

[21] X. Y. Qin, H. T. Wu, C. Cao, Y. P. Loh, and Y. Cheng, “A metaanalysis of peripheral blood nerve growth factor levels in patients with schizophrenia," Molecular Psychiatry, vol. 22, no. 9, pp. 1306-1312, 2017.

[22] R. M. Ahmed, E. Highton-Williamson, J. Caga et al., "Lipid metabolism and survival across the frontotemporal dementia-amyotrophic lateral sclerosis spectrum: relationships to eating behavior and cognition," Journal of Alzheimer's Disease, vol. 61, no. 2, pp. 773-783, 2018.

[23] A. N. A. B. Barros, M. E. T. Dourado, L. F. C. Pedrosa, and L. Leite-Lais, "Association of copper status with lipid profile and functional status in patients with amyotrophic lateral sclerosis," Journal of Nutrition and Metabolism, vol. 2018, Article ID 5678698, 7 pages, 2018.

[24] M. C. Boll, L. Bayliss, S. Vargas-Canas et al., "Clinical and biological changes under treatment with lithium carbonate and valproic acid in sporadic amyotrophic lateral sclerosis," Journal of the Neurological Sciences, vol. 340, no. 1-2, pp. 103-108, 2014.

[25] D. Bonnefont-Rousselot, L. Lacomblez, M. C. Jaudon et al., "Blood oxidative stress in amyotrophic lateral sclerosis," Journal of the Neurological Sciences, vol. 178, no. 1, pp. 57-62, 2000.

[26] A. Chio, A. Calvo, A. Ilardi et al., "Lower serum lipid levels are related to respiratory impairment in patients with ALS," Neurology, vol. 73, no. 20, pp. 1681-1685, 2009.

[27] A. Conti, S. Iannaccone, B. Sferrazza et al., "Differential expression of ceruloplasmin isoforms in the cerebrospinal fluid of amyotrophic lateral sclerosis patients," Proteomics Clinical Applications, vol. 2, no. 12, pp. 1628-1637, 2008.

[28] S. De Benedetti, G. Lucchini, C. Del Bò et al., "Blood trace metals in a sporadic amyotrophic lateral sclerosis geographical cluster," BioMetals, vol. 30, no. 3, pp. 355-365, 2017.

[29] J. B. Delaye, F. Patin, E. Piver et al., "Low IDL-B and high LDL-1 subfraction levels in serum of ALS patients," Journal of the Neurological Sciences, vol. 380, pp. 124-127, 2017.

[30] L. Dupuis, P. Corcia, A. Fergani et al., "Dyslipidemia is a protective factor in amyotrophic lateral sclerosis," Neurology, vol. 70, no. 13, pp. 1004-1009, 2008.

[31] R. Huang, X. Guo, X. Chen et al., "The serum lipid profiles of amyotrophic lateral sclerosis patients: a study from south-west China and a meta-analysis," Amyotrophic Lateral Sclerosis and Frontotemporal Degeneration, vol. 16, no. 5-6, pp. 359-365, 2015.

[32] Y. Ihara, K. Nobukuni, H. Takata, and T. Hayabara, "Oxidative stress and metal content in blood and cerebrospinal fluid of amyotrophic lateral sclerosis patients with and without a $\mathrm{Cu}$, Zn-superoxide dismutase mutation," Neurological Research, vol. 27, no. 1, pp. 105-108, 2005.

[33] K. Ikeda, T. Hirayama, T. Takazawa, K. Kawabe, and Y. Iwasaki, "Relationships between disease progression and serum levels of lipid, urate, creatinine and ferritin in Japanese patients with amyotrophic lateral sclerosis: a cross-sectional study," Internal Medicine, vol. 51, no. 12, pp. 1501-1508, 2012.

[34] G. D. Kanias and E. Kapaki, "Trace elements, age, and sex in amyotrophic lateral sclerosis disease," Biological Trace Element Research, vol. 56, no. 2, pp. 187-201, 1997.
[35] D. Keizman, M. Ish-Shalom, S. Berliner et al., "Low uric acid levels in serum of patients with ALS: further evidence for oxidative stress?," Journal of the Neurological Sciences, vol. 285, no. 1-2, pp. 95-99, 2009.

[36] T. Kihira, K. Okamoto, S. Yoshida et al., "Environmental characteristics and oxidative stress of inhabitants and patients with amyotrophic lateral sclerosis in a high-incidence area on the Kii Peninsula, Japan," Internal Medicine, vol. 52, no. 13, pp. 1479-1486, 2013.

[37] S. M. Kim, M. Y. Noh, H. Kim et al., "25-Hydroxycholesterol is involved in the pathogenesis of amyotrophic lateral sclerosis," Oncotarget, vol. 8, no. 7, pp. 11855-11867, 2017.

[38] A. LoGerfo, L. Chico, L. Borgia et al., "Lack of association between nuclear factor erythroid-derived 2-like 2 promoter gene polymorphisms and oxidative stress biomarkers in amyotrophic lateral sclerosis patients," Oxidative Medicine and Cellular Longevity, vol. 2014, Article ID 432626, 9 pages, 2014.

[39] R. M. Mitchell, Z. Simmons, J. L. Beard, H. E. Stephens, and J. R. Connor, "Plasma biomarkers associated with ALS and their relationship to iron homeostasis," Muscle \& Nerve, vol. 42, no. 1, pp. 95-103, 2010.

[40] K. Mokuno, K. Kiyosawa, H. Honda et al., "Elevated serum levels of manganese superoxide dismutase in polymyositis and dermatomyositis," Neurology, vol. 46, no. 5, pp. 1445-1447, 1996.

[41] J. A. Molina, F. de Bustos, F. J. Jiménez-Jiménez et al., "Serum levels of coenzyme $\mathrm{Q}_{10}$ in patients with amyotrophic lateral sclerosis," Journal of Neural Transmission, vol. 107, no. 8-9, pp. 1021-1026, 2000.

[42] Y. Nadjar, P. Gordon, P. Corcia et al., "Elevated serum ferritin is associated with reduced survival in amyotrophic lateral sclerosis," PLoS One, vol. 7, no. 9, article e45034, 2012.

[43] M. Nagase, Y. Yamamoto, Y. Miyazaki, and H. Yoshino, "Increased oxidative stress in patients with amyotrophic lateral sclerosis and the effect of edaravone administration," Redox Report, vol. 21, no. 3, pp. 104-112, 2016.

[44] S. I. Oh, S. Baek, J. S. Park, L. Piao, K. W. Oh, and S. H. Kim, "Prognostic role of serum levels of uric acid in amyotrophic lateral sclerosis," Journal of Clinical Neurology, vol. 11, no. 4, pp. 376-382, 2015.

[45] P. I. Oteiza, O. D. Uchitel, F. Carrasquedo, A. L. Dubrovski, J. C. Roma, and C. G. Fraga, "Evaluation of antioxidants, protein, and lipid oxidation products in blood from sporadic amyotrophic lateral sclerosis patients," Neurochemical Research, vol. 22, no. 4, pp. 535-539, 1997.

[46] A. Pasquinelli, L. Chico, L. Pasquali et al., "Gly482Ser PGC- $1 \alpha$ gene polymorphism and exercise-related oxidative stress in amyotrophic lateral sclerosis patients," Frontiers in Cellular Neuroscience, vol. 10, p. 102, 2016.

[47] T. L. Peters, J. D. Beard, D. M. Umbach et al., "Blood levels of trace metals and amyotrophic lateral sclerosis," NeuroToxicology, vol. 54, pp. 119-126, 2016.

[48] A. Radunović, H. T. Delves, W. Robberecht et al., "Copper and zinc levels in familial amyotrophic lateral sclerosis patients with CuZnSOD gene mutations," Annals of Neurology, vol. 42, no. 1, pp. 130-131, 1997.

[49] N. A. Sutedja, Y. T. van der Schouw, K. Fischer et al., "Beneficial vascular risk profile is associated with amyotrophic lateral sclerosis," Journal of Neurology, Neurosurgery \& Psychiatry, vol. 82, no. 6, pp. 638-642, 2011. 
[50] G. Torsdottir, J. Kristinsson, G. Gudmundsson, J. Snaedal, and T. Johannesson, "Copper, ceruloplasmin and superoxide dismutase (SOD) in amyotrophic lateral sclerosis," Pharmacology and Toxicology, vol. 87, no. 3, pp. 126-130, 2000.

[51] C. Veyrat-Durebex, P. Corcia, A. Mucha et al., "Iron metabolism disturbance in a French cohort of ALS patients," BioMed Research International, vol. 2014, Article ID 485723, 6 pages, 2014.

[52] A. Wuolikainen, J. Acimovic, A. Lovgren-Sandblom, P. Parini, P. M. Andersen, and I. Bjorkhem, "Cholesterol, oxysterol, triglyceride, and coenzyme Q homeostasis in ALS. Evidence against the hypothesis that elevated 27-hydroxycholesterol is a pathogenic factor," PLoS One, vol. 9, no. 11, article e113619, 2014.

[53] J. W. Yang, S. M. Kim, H. J. Kim et al., "Hypolipidemia in patients with amyotrophic lateral sclerosis: a possible gender difference?," Journal of Clinical Neurology, vol. 9, no. 2, pp. 125-129, 2013.

[54] Y. Zheng, L. Gao, D. Wang, and D. Zang, "Elevated levels of ferritin in the cerebrospinal fluid of amyotrophic lateral sclerosis patients," Acta Neurologica Scandinavica, vol. 136, no. 2, pp. 145-150, 2017.

[55] Z. Zheng, X. Guo, Q. Wei et al., "Serum uric acid level is associated with the prevalence but not with survival of amyotrophic lateral sclerosis in a Chinese population," Metabolic Brain Disease, vol. 29, no. 3, pp. 771-775, 2014.

[56] S. Zoccolella, I. L. Simone, R. Capozzo et al., “An exploratory study of serum urate levels in patients with amyotrophic lateral sclerosis," Journal of Neurology, vol. 258, no. 2, pp. 238-243, 2011.

[57] E. D’Amico, P. Factor-Litvak, R. M. Santella, and H. Mitsumoto, "Clinical perspective on oxidative stress in sporadic amyotrophic lateral sclerosis," Free Radical Biology and Medicine, vol. 65, pp. 509-527, 2013.

[58] F. Bozzo, A. Mirra, and M. T. Carrì, "Oxidative stress and mitochondrial damage in the pathogenesis of ALS: New perspectives," Neuroscience Letters, vol. 636, pp. 3-8, 2017.

[59] G. Djordjevic, S. Ljubisavljevic, S. Sretenovic, G. Kocic, I. Stojanovic, and S. Stojanovic, "The cerebrospinal fluid values of advanced oxidation protein products and total thiol content in patients with amyotrophic lateral sclerosis," Clinical Neurology and Neurosurgery, vol. 163, pp. 33-38, 2017.

[60] T. Murata, C. Ohtsuka, and Y. Terayama, "Increased mitochondrial oxidative damage and oxidative DNA damage contributes to the neurodegenerative process in sporadic amyotrophic lateral sclerosis," Free Radical Research, vol. 42, no. 3, pp. 221-225, 2008.

[61] Z. Pu, W. Xu, Y. Lin, J. He, and M. Huang, "Oxidative stress markers and metal ions are correlated with cognitive function in Alzheimer's disease," American Journal of Alzheimer's Disease \& Other Dementias ${ }^{\circledR}$, vol. 32, no. 6, pp. 353-359, 2017.

[62] A. Bolner, R. Micciolo, O. Bosello, and G. P. Nordera, "A panel of oxidative stress markers in Parkinson's disease," Clinical Laboratory, vol. 62, no. 1-2, pp. 105-112, 2016.

[63] M. Schrag, C. Mueller, M. Zabel et al., "Oxidative stress in blood in Alzheimer's disease and mild cognitive impairment: a meta-analysis," Neurobiology of Disease, vol. 59, pp. 100-110, 2013.

[64] Z. Wei, X. Li, X. Li, Q. Liu, and Y. Cheng, "Oxidative stress in Parkinson's disease: a systematic review and meta-analysis," Frontiers in Molecular Neuroscience, vol. 11, p. 236, 2018. 


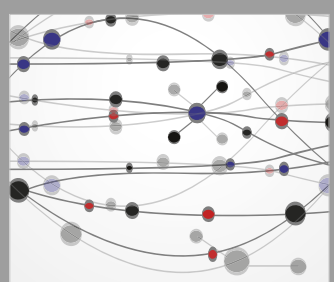

The Scientific World Journal
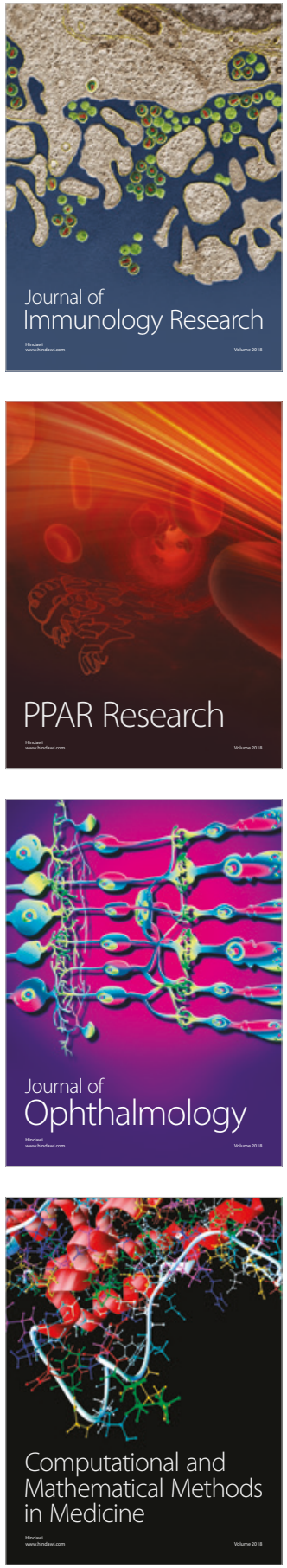

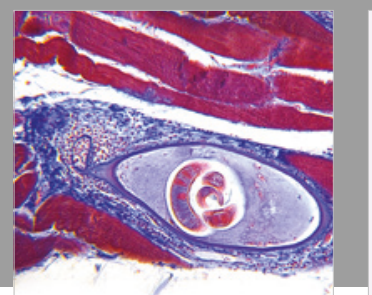

Gastroenterology Research and Practice

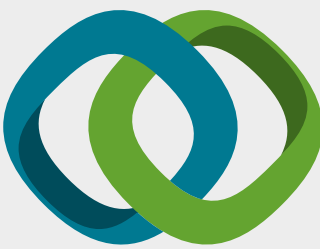

\section{Hindawi}

Submit your manuscripts at

www.hindawi.com
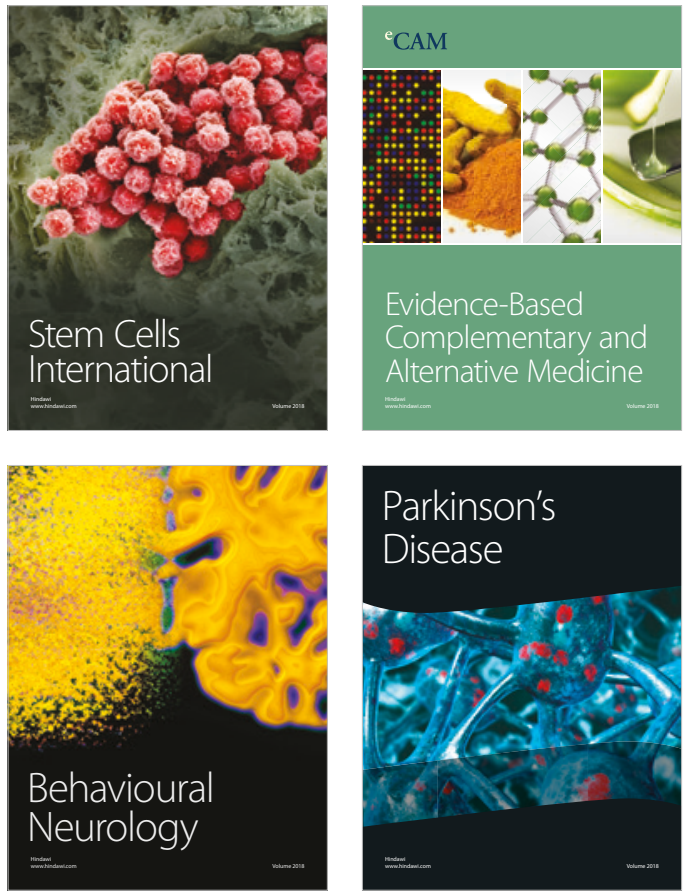

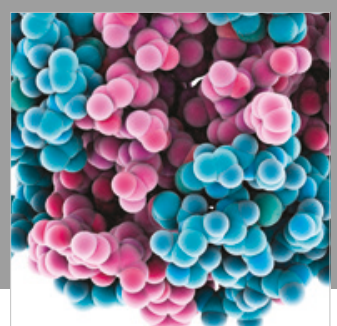

ournal of

Diabetes Research

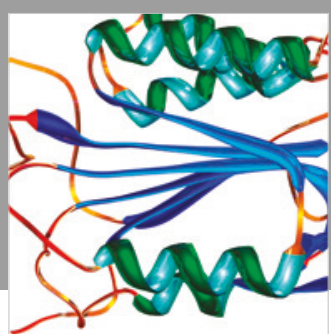

Disease Markers
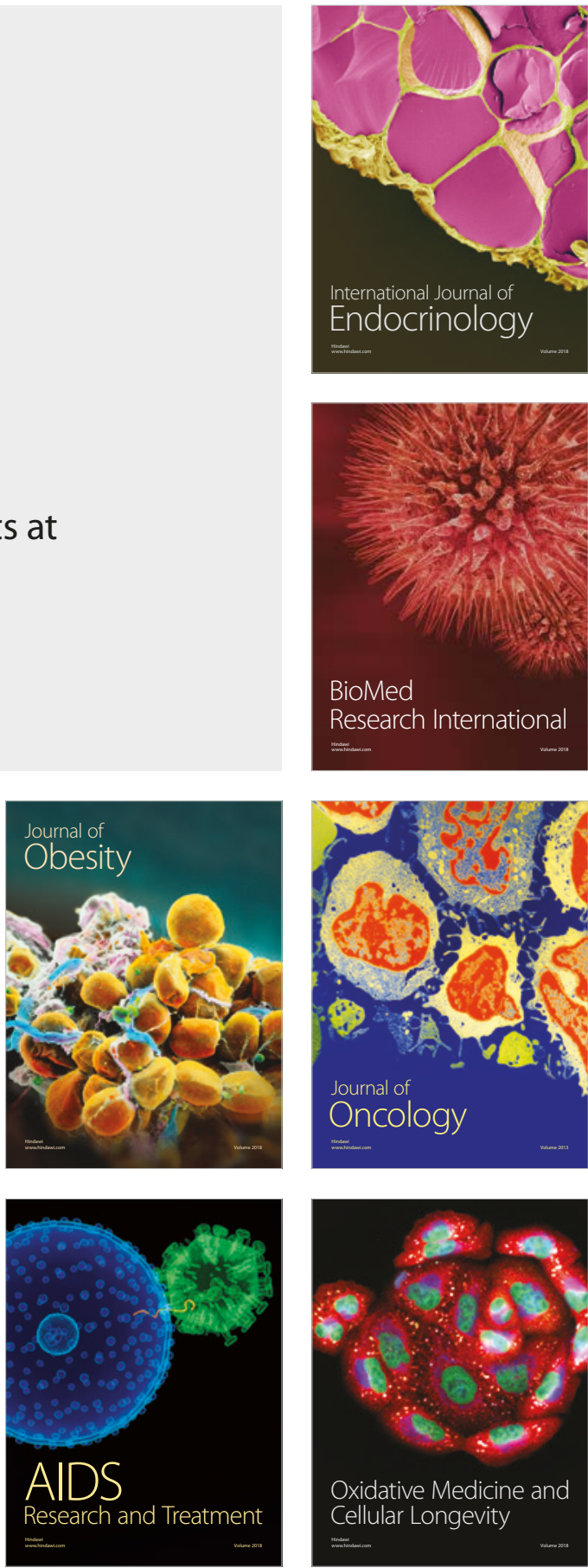